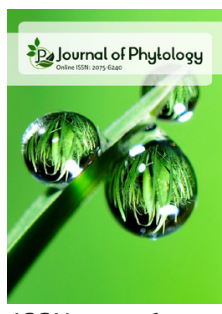

ISSN: $2075-6240$

\title{
An in silico approach towards exploration of the oxidative stress resistance of major withanolides of Withania somnifera in relation to COVID-19 management
}

\author{
Sangeetha Vinodkumar, Krishnapriya Santhanu, Kanimozhi Natarajan, \\ Kalaiselvi Senthil*
}

Department of Biochemistry, Biotechnology and Bioinformatics, Avinashilingam Institute of Home Science and Higher Education for Women, Coimbatore- 641043, Tamil Nadu, India

\begin{abstract}
Oxidative stress is the state of imbalance between the production of reactive oxygen species (free radicals) in the biological system and the ability of the body to detoxify them resulting in increased accumulation of free radicals in the cells. This stress leads to weakening of the immune system thus leading to higher susceptibility to other infections as well. This also includes the weakening of the respiratory tract leading to increased susceptibility of viral infections as in the case of COVID-19. Treatment for any kind of abnormality requires the identification of the key target proteins and pathways that are being altered. Withania somnifera is being used in the traditional medicinal system to improve health and longevity thus creating a sense of mental as well as physical well being. The present study utilises network pharmacological approach to predict the potential oxidative stress targets of the three major withanolides: withanolide A, withaferin A and withanone. Primarily, the targets of the individual withanolides were obtained from the Swiss target and DIGEP-pred databases and the GO terms and lead hits related to oxidative stress were retrieved from AMIGO2 database. Totally 40 correlative hits were obtained as anti stress targets of the withanolides, which were subjected to functional enrichment and protein-protein interaction analysis to study the enriched pathways underlying oxidative stress response. Further the eleven crucial targets of the four selected pathways were analysed using molecular docking analysis. A total of forty protein hits were obtained as oxidative stress targets of the withanolides. Further, the pathway enrichment of these forty target genes showed the AGE RAGE signalling pathway as highly enriched pathway. Therefore, the AGE RAGE signalling pathway along with its underlying pathways namely MAPK signalling pathway, FOXO pathway and PI3-AKT pathway were chosen among all the other enriched pathways. Further the molecular docking analysis of the eleven target proteins falling under these four pathways showed good docking scores of the withanolides with all the eleven targets with highest interaction against BCL2. From the above study, the biological targets and associated pathways of the withanolides have been retrieved. Thus the in silico approach undertaken in this study explores the role of the key withanolides in the antioxidant potential of the traditional medicinal plant Withania somnifera.
\end{abstract}

*Corresponding authors:

Kalaiselvi Senthil

E-mail: kalaiselvi_bc@avinuty.ac.in KEYWORDS: Withaferin a, Oxidative stress, Pathway enrichment, Amigo2, Target prediction, Protein-protein interaction.

\section{INTRODUCTION}

Oxidative stress (OS) is a condition of increased accumulation of reactive oxygen species (ROS) in the body. It is caused by the imbalance in the generation and scavenging of ROS in cells and tissues due to the inability of the biological system to detoxify them. Increased levels of ROS results in the oxidation of nucleic acids, proteins and lipids leading to irreversible damage to the cellular structures (Farías et al., 2017). Oxidative stress, directly or indirectly serves to influence the activation or deactivation of multiple signalling pathways. Thus OS plays an important role in the susceptibility of many chronic diseases (Prasad \& Srivastava, 2020) like atherosclerosis (Matsuoka., 2001), renal disorders (Pawlak et al., 2009), retinal diseases (Masuda et al., 2017), cardiovascular problems (Farías et al., 2017), oral cancer (Yu et al., 2020), male infertility (Ahmad et al., 2010), neurodegenerative disorders (Shah et al., 2015) and a plethora of other diseases. As there exists a pathway crosstalk between COVID-19 and most of these comorbid conditions, they also lead to an increase in severity of COVID-19

\footnotetext{
Copyright: $\odot$ The authors. This article is open access and licensed under the terms of the Creative Commons Attribution License (http://creativecommons.org/licenses/by/4.0/) which permits unrestricted, use, distribution and reproduction in any medium, or format for any purpose, even commercially provided the work is properly cited. Attribution - You must give appropriate credit, provide a link to the license, and indicate if changes were made.
} 
infection (Chakrabarty et al., 2021). Also the treatment of COVID-19 patients with these comorbidities becomes more complex as there is no drug-drug interaction assessment studies with these comorbidity pathways (Barh et al., 2021). Further the increased cytokine production as a immune response against infections also serves as a source of ROS production which in turn can lead to increased viral replication (Bakadia et al., 2020). This increase in ROS levels is also accompanied by significant decrease in levels of vitamin A, C and $\mathrm{E}$ in plasma along with diminished antioxidant enzyme activities in COVID-19 patients (Muhammad et al., 2021). This in turn leads to cell apoptosis mediated by increased cytokine production as reported in severe COVID-19 cases. Thus oxidative stress plays important role in pathogenesis of COVID-19 infection (Chernyak et al., 2020).

Since several decades medicinal plants have been in use for the control and treatment of various clinical conditions. Further current researches focus more on these traditional medicinal plants which have been a cost effective and healthy source for the control of oxidative stress and prevention of OS related disorders (Palipoch \& Thammarat, 2013). Medicinal plants and herbal extracts with natural antioxidants could potentially boost the body to defend against oxidative stress and thus influence the immune pathways to act against various diseased conditions. Withania somnifera (ashwagandha) as mentioned in Indian Pharmacopoeia (1985) is categorised as rasayan in Indian traditional medicinal system. This plant is reported in various studies to defend the body against various environmental factors, revitalise the body and thus increase longevity (Bhattacharya \& Muruganandam, 2003). Recently, during this covid-19 pandemic, this magic herb has been promoted by the Indian government for use as an immune booster. Besides acting directly as an immune enhancer, it also helps to overcome any alterations in the immune activity caused by the psychological distress, anxiety and depression due the pandemic (Rajkumar, 2020). Thus Ws is accurately referred to as an adaptogenic drug.

Withanolides, the bioactive compounds from Withania somnifera, are studied worldwide for its various therapeutic activities. They are reported to regulate oxidative stress and cytokine secretion (Balakrishna et al., 2021). Withaferin A is the most important and the first withanolide to be reported as early as 1962 . Since then, nearly 400 closely related compounds have been studied under the class 'withanolides'. Since the epigenetic mechanism and genes underlying oxidative stress overlaps with that of aging process, withaferin A that showed good resistance against oxidative stress also showed increase in lifespan when added as a feed in studies using drosophila(Koval et al., 2021). Withania somnifera extracts and its bioactive component withanone are found to control oxidative stress in neuroblastoma and glioblastoma cell lines thus protecting against neurodegeneration (Shah et al., 2015). Withanolide A has also reduced the ROS levels and thus increased lifespan in various strains of C. elegans (Nab et al., 2021). Further, in the molecular docking studies against the M-pro protein (the potential therapeutic target of COVID-19), withaferin A exhibited good binding affinity of $-7.7 \mathrm{Kcal} / \mathrm{mol}$ among a pool of FDA approved anti-viral compounds and phytochemicals studied (Chandel et al., 2020). This interaction of withaferin
A with M-pro was found to be much stronger than the other repurposed drugs including hydroxychloroquine (Dhawan et al., 2021). Withanone also showed good docking potential similar to N3 inhibitor towards M-pro. Withanone was found to form polar bonds with Cys-145 residue of M-pro, which is important for its catalytic activity (Kumar et al., 2020). Thus all these three prime withanolides, withanolide $\mathrm{A}$, withanone and withaferin $\mathrm{A}$ are known for its effect against oxidative stress and ROS accumulation along with increasing the well being and life span of the organisms. Hence all these three magical compounds can be used as adaptogenic or geroprotective drug.

Withania somnifera extracts as well as individual withanolides have been reported for various bioactivities along with their effect on a few genes associated with the respective disease. But, the complete set of targets that are acted upon by these withanolides have not been elucidated. Further, ashwagandha being an adaptogenic herb effective against various disorders it is necessary to understand its key targets. Since oxidative stress is one among the major factors of most of the disease worldwide, the exploration of the target proteins behind the antioxidant potential of this herb is essential. Further since oxidative stress is also reported to increase the comorbidities in COVID-19, it is also essential to understand the role of OS related genes and pathways on COVID-19. Therefore the current study is novel in that it aims to identify the potential targets of these three important withanolides and make use of network pharmacology approach to establish the various pathways altered by these withanolides to combat oxidative stress condition.

\section{EXPERIMENTAL MATERIALS AND METHODS}

\section{Structure Retrieval and Pharmacological Profiling}

The 3D structure in SDF format and canonical SMILES of the ligands withaferin A (Pubchem CID: 265237), withanolide A (Pubchem CID: 11294368) and Withanone (Pubchem CID: 21679027) were retrieved from PubChem database (https:// pubchem.ncbi.nlm.nih.gov/). The SDF format file was then converted to PDB format using Open Babel GUI. The canonical SMILES was used as input files for Swiss ADME (http:// www.swissadme.ch/), a freely accessible web tool to study the Adsorption, Distribution, Metabolism, Excretion and Toxicity (ADMET) profile.

\section{Prediction of Oxidative Stress Targets of Withanolides}

Two freely accessible target protein databases "Swiss target" (http://www.swisstargetprediction.ch/) (Daina et al., 2019) and "DIGEP-pred" (http://www.way2drug.com/ge/) (Khanal et al., 2020) were used to predict the biological targets of each ligand with biological activity $(\mathrm{Pa})>0.5$. The canonical SMILES were used as the query file for both the databases. The target sets from both the databases were then pooled to get the final targets of each withanolide. Following this, the target hits related to oxidative stress were extracted from AmiGO2 version 2.1.15 (http://amigo.geneontology.org/) (Carbon et al., 2009) database using the key search term 'oxidative stress'. A total of nine 
AmiGO 2 gene ontology terms related to oxidative stress were chosen. All the nine target GO data sets were combined and duplicates removed to obtain the final list of oxidative stress related target proteins. Finally the targets of the individual withanolides overlapping with the targets of oxidative stress were retrieved by constructing venn diagrams in FunRich software (Fonseka et al., 2021). The gene set obtained were considered as anti-stress targets of that particular withanolide. The GO analysis of the obtained target hits were performed using Funrich software under three categories: cellular component, molecular function and biological process. Each of the enriched GO terms was plotted as graphs of the top 10 enriched terms.

\section{Protein Protein Interaction (ppi) Network Construction}

The final screened oxidative stress targets of the individual withanolides were pooled and protein set containing 40 entries was extracted and queried on STRING (https://string-db.org/) for Homo sapiens (Szklarczyk et al., 2019). The protein protein interaction network was used to observe the protein to protein co-relation and their pathway enrichment based on confidence scoring. The pathway enrichment of the genes was based on the KEGG pathway database. Among the enriched pathways, the pathways modulated by oxidative stress as obtained from published literature were chosen. The proteins of the highly enriched pathway AGE RAGE signalling pathway along with its underlying pathways namely FOXO signalling pathway, AKT signalling pathway and MAPK signalling pathway were selected for further analysis.

\section{Molecular Docking}

The predicted targets from the network analysis were then evaluated by molecular docking. The 3D structures of the eleven protein targets obtained from the selected four pathways: FOXOl(PDB ID: 3COA), MDM2 (PDB ID: 5AFG), JUN (PDB ID: 1JUN), BCL2 (PDB ID: 6QGG), SYK (PDB ID: 4XG2), PDGFRB (PDB ID: 2L6W), MAPKI (PDB ID: 6G54), MAPK8 (PDB ID: 3O2M), MAPK14 (PDB ID: 7BDQ), PRKCD (PDB ID: 1PTR) and JAK2 (PDB ID: 5WIJ) were retrieved from protein data bank (PDB) (https://www.rcsb.org/). The water molecules and attached ligands were removed using Pymol 2. Further the proteins were prepared by addition of charges and polar hydrogen atoms using Autodock tools. Each of these protein targets were then docked against withaferin A, withanolide A and withanone individually using Autodock 1.5.6. Both the protein and the ligand were considered as rigid during the docking process. The binding pose with the lowest binding energy among the ten different confirmations were extracted as the best docking pose. The docked files were then visualized using Discovery studio Biovia 2020 to study the binding pockets and the interacting residues.

\section{RESULTS}

\section{Structure Retrieval and Adme Properties of Withanolides}

The 3D structure and canonical SMILES of the three chosen ligands were retrieved from Pubchem Database (Figure 1).
The ADME properties in terms of physicochemical properties, lipophilicity, water solubility, pharmacokinetics, druglikeness and medicinal chemistry were analysed (Table 1) using Swiss ADME. All the three withanolides being structurally similar, their physicochemical properties were found to be relatively same with molecular weight $(470.606 \mathrm{~g} / \mathrm{mol})$, six hydrogen acceptors and two hydrogen donors which were all within the permissible limits for a drug compound. All the three ligands showed no violations against the lipinski's rule of 5 .

Highly soluble compounds are highly absorbed. The solubility and lipophilicity of a drug compound is expressed in terms of $\log \mathrm{S}$ mol/L and $\log \mathrm{p}$ respectively. The absorption and distribution of the drug molecule is dependent on its aqueous solubility. The drugs with Log $\mathrm{S}$ values between -1 and -4 are considered optimum for better absorption and distribution (Lohidashan et al., 2018). The lower the log P values, stronger are the lipophilicity of the compound. Swiss ADME predicts the hydrophilic and lipophilic nature of compounds in terms of log $\mathrm{P}$ and $\log \mathrm{S}$ programs like ILOGP, XLOGP3, WLOGP, ESOL and SILICOS-IT. The three compounds tested were found to be moderately soluble with $\log \mathrm{S}$ values $-4.67,-4.97,-4.56$ for withanolide $\mathrm{A}$, withaferin $\mathrm{A}$ and withanone respectively.

The metabolic capability of the drug compound is an important factor to be considered in drug discovery. The cytochrome $\mathrm{P} 450$, is a group of enzyme capable of metabolising a variety of xenobiotics. Of the 57 cytochrome P450s (CYPs) isoforms in humans, only five isoforms CYP3A4, CYP2D6, CYP2C19, CYP2C9 and CYP1A2 are responsible for about $90 \%$ of the drug metabolism (Aswathy et al., 2018). Withaferin A, withanolide A and withanone showed no inhibition to all the five isoforms of cyp 450s. All the three ligands showed bioavailability score of 0.55 . The skin permeation values were found to be around $-6 \mathrm{~cm} / \mathrm{s}$ and synthetic accessibility score around 6. All these pharmacokinetic properties are within the permissible range described for human use, indicating its potential as a drug-like molecule.

Table 1: Physicochemical, pharmacokinetic and drug likeliness profile of the bioactive compounds

\begin{tabular}{|c|c|c|c|}
\hline Compound name & $\begin{array}{c}\text { Withanolide } \\
\text { A }\end{array}$ & Withaferin $\mathrm{A}$ & Withanone \\
\hline Molecular weight $(\mathrm{g} / \mathrm{mol})$ & 470.60 & 470.66 & 470.60 \\
\hline No: heavy atoms & 34 & 34 & 34 \\
\hline No: hydrogen bond acceptors & 6 & 6 & 6 \\
\hline No: hydrogen bond donos & 2 & 2 & 2 \\
\hline No: rotatable bonds & 2 & 3 & 2 \\
\hline Polar surface area $\left(\AA^{2}\right)$ & 96.36 & 96.36 & 96.36 \\
\hline $\log P(i L O G P)$ & 3.68 & 3.39 & 3.71 \\
\hline $\log S(E S O L)$ & -4.67 & -4.97 & -4.56 \\
\hline GI absorption & High & High & High \\
\hline $\begin{array}{l}\text { CYP (1A2, 2C19, 2C9, 2D6, } \\
3 A 4) \text { inhibitor }\end{array}$ & No & No & No \\
\hline Skin permeation $\operatorname{LogK}_{p} \mathrm{~cm} / \mathrm{s}$ & -6.86 & -6.45 & -7.01 \\
\hline Bioavailability score & 0.55 & 0.55 & 0.55 \\
\hline Synthetic accessibility & 6.39 & 6.83 & 6.38 \\
\hline Lipinski (No: of violations) & 0 & 0 & 0 \\
\hline Ghose (No: of violations) & 1 (atoms $>70$ ) & 1 (atoms $>70$ ) & 1 (atoms $>70$ ) \\
\hline Veber (No: of violations) & 0 & 0 & 0 \\
\hline Egan (No: of violations) & 0 & 0 & 0 \\
\hline Muegge (No: of violations) & 0 & 0 & 0 \\
\hline
\end{tabular}

J Phytol • $2021 \quad \bullet \quad \mathrm{Vol} 13$ 


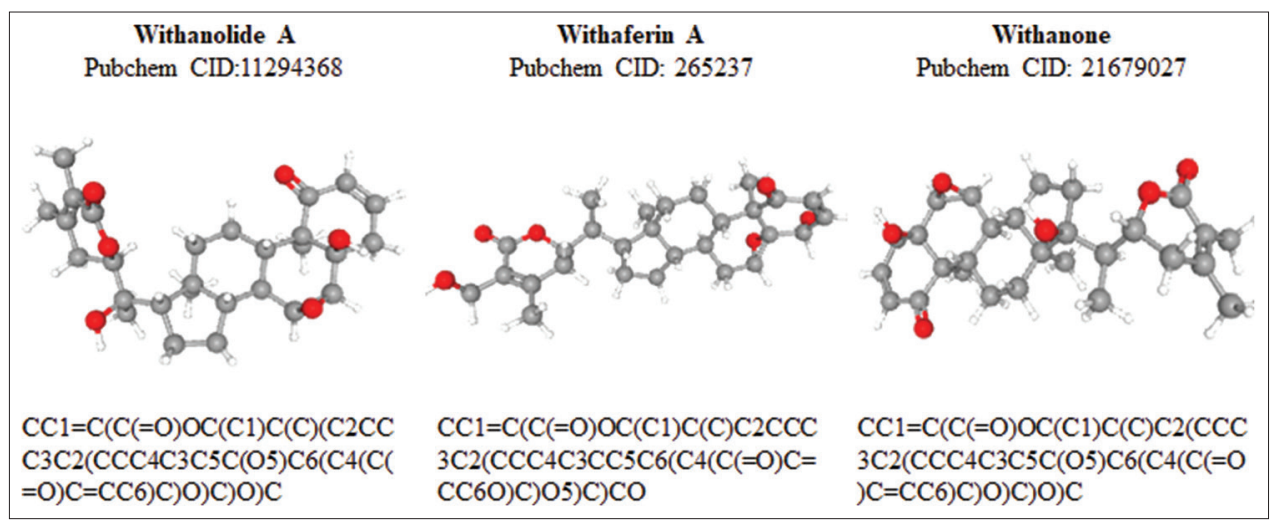

Figure 1: 3D Structure and Canonical SMILES of withaferin A, withanolide $A$ and withanone

\section{Screening and Enrichment Analysis of Oxidative Stress Responsive Targets of Withanolides}

The targets for withaferin A, withanolide A and withanone were obtained from Swiss target and DIGEP-pred platforms using their respective canonical SMILES file as an input. The targets from both the databases were then merged and duplicates removed. The final number of target proteins for each series obtained is presented table 2 . The highest number of target genes were obtained for withanolide A with a total of 317 targets followed by 289 and 273 targets for withanone and withaferin A respectively.

AmiGO 2 is an online free interactive gene ontology and annotation browser that helps to navigate and filter data from any specific ontology branch. The target genes underlaying oxidative stress response were obtained from AmiGO 2 database using 'oxidative stress' as the search term. A total of nine gene ontology terms related to oxidative stress were selected and protein targets retrieved. All the data were then pooled to yield a final list of 572 genes responsible in stress response (Table 3).

The protein targets that were common between the withanolides and the oxidative stress related targets were interpreted by constructing venn diagrams using Funrich software. The common genes were considered as the oxidative stress targets of that withanolide. A total of 30, 27 and 31 oxidative stress target proteins were obtained for withanolide A, withaferin $\mathrm{A}$ and withanone respectively. Totally the 40 oxidative stress target hits of the withanolides were subjected to Gene Ontology (GO) enrichment analysis based on three categories: cellular component, molecular function and biological process using Funrich software. The top six GO terms that were significantly enriched among the 40 core targets were retrieved. The enrichment analysis on cellular component shows that highly significant GO terms were associated with the nucleus and cytoplasm (Figure 2a). Figure 2b shows the enriched GO terms of molecular function were protein serine/threonine kinase activity, protein-tyrosine kinase activity and catalytic activity. Among the biological processes, signal transduction and cell communication GO terms were found to be highly enriched (Figure 2c).
Table 2: Target proteins of the withanolides

\begin{tabular}{lccc}
\hline Compound & Swiss target & DIGEP-pred & Total \\
\hline Withanolide A & 106 & 217 & 317 \\
Withaferin A & 102 & 174 & 273 \\
Withanone & 113 & 186 & 289 \\
\hline
\end{tabular}

Table 3: 0xidative stress targets retrieved under each gene ontology term

\begin{tabular}{llc} 
G0 Accession & Pathway & $\begin{array}{c}\text { No: of } \\
\text { po: }\end{array}$ \\
\hline G0: 0000302 & Response to reactive oxygen species \\
G0: 0034614 & Cellular response to reactive oxygen species & 222 \\
G0: 0072593 & Reactive oxygen species metabolic process & 239 \\
G0: 2000377 & Regulation of R0S metabolic process & 157 \\
G0: 1901031 & Regulation of response to reactive oxygen & 040 \\
& species & \\
G0: 0006979 & Response to oxidative stress & 456 \\
G0: 0034599 & Cellular response to oxidative stress & 305 \\
G0: 1902882 & Regulation of response to oxidative stress & 101 \\
G0: 1900407 & Regulation of cellular response to oxidative & 118 \\
& stress &
\end{tabular}

\section{Protein-Protein Interaction Network and Pathway Enrichment Analysis}

The protein-protein interaction network for this target set of 40 proteins was constructed using STRING. The interaction network constituted of 125 edges for 40 nodes with average node degree of 6.25 and enrichment value of $<1.0 \mathrm{e}-16$. The pathway enrichment was based on KEGG pathway database in which a total of 112 pathways were found to be enriched. This included various pathways related to infectious diseases (tuberculosis, eipstein-barr virus infection, leishmaniasis, toxoplasmosis, salmonella infection, shigellosis, influenza A), pathways influencing cancer (pathways in cancer, renal cell carcinoma, pancreatic cancer, microRNAs in cancer, prostrate cancer, transcriptional misregulation in cancer, bladder cancer, apoptosis), diabetes (glucagon signalling pathway, typeII diabetes mellitus, insulin signalling pathway) and signalling pathways (neurotropin signalling pathway, GnRH signalling pathway, ErbB signalling pathway, prolactin signalling pathway, NOD like receptor signalling pathway, VEGF signalling pathway, chemokine signalling pathway). Among these enriched 


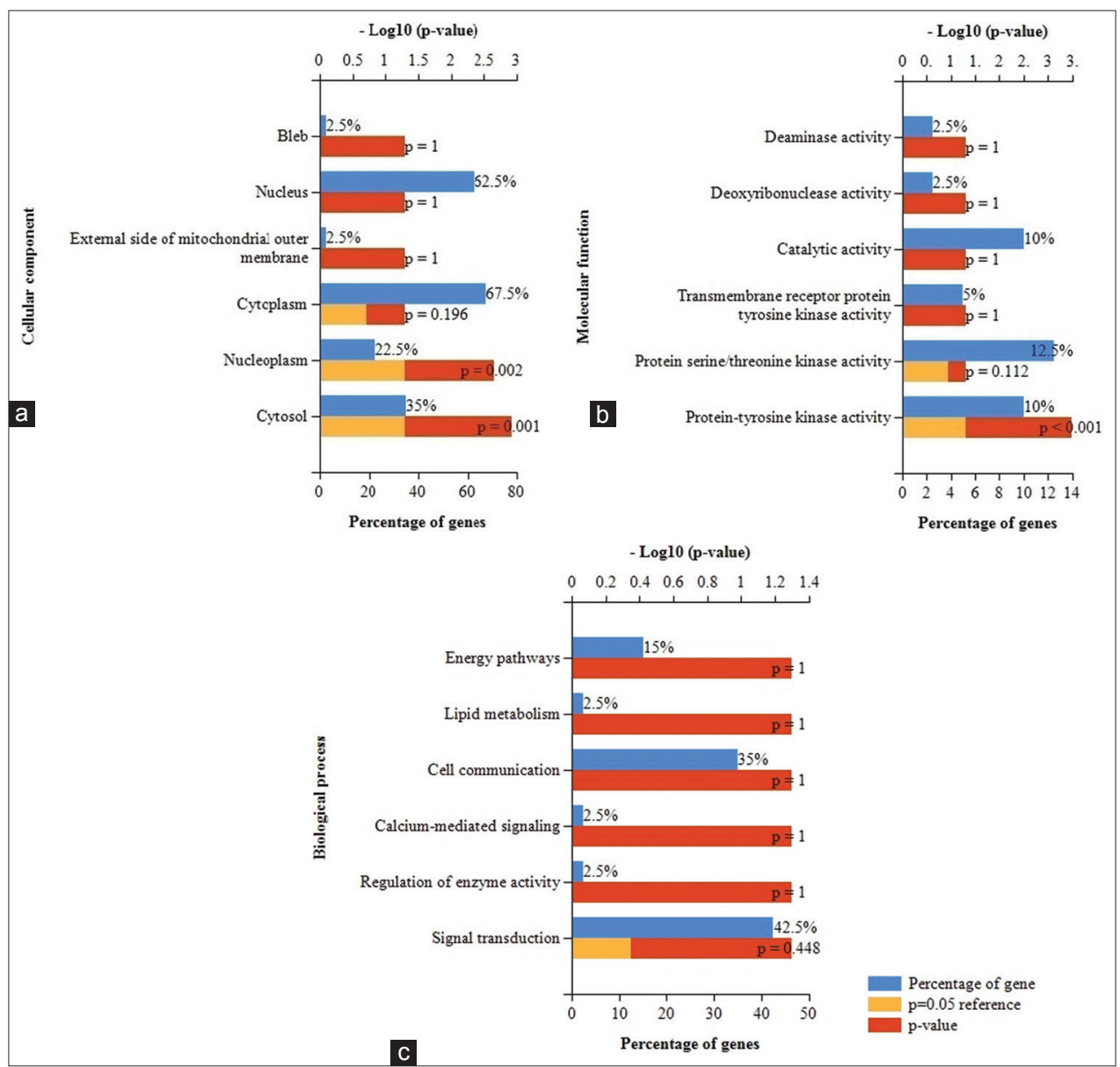

Figure 2: Gene ontology enrichment analysis of the potential oxidative stress targets of withanolides *Gene ontology of the screened list of proteins in terms of a) cellular component b) molecular function and c) biological process

pathways, only those pathways related to oxidative stress were chosen for further studies.

Thus the KEGG pathways chosen were AGE-RAGE signalling pathway, MAPK signalling pathway, FOXO signalling pathway and P13-AKT signalling pathway. Among the oxidative stress pathways chosen, AGE-RAGE signalling pathway was highly enriched with false discovery rate of 6.81e-09, followed by FOXO signalling pathway, P13 AKT signalling pathway and MAPK signalling pathway with false discovery rate of 4.32e- $05,0.00024$ and 0.00087 respectively. A total of eleven target proteins out of the forty targets of the withanolides fell under these chosen pathways. The interplay between these four selected pathways in controlling ROS accumulation is depicted in Figure 3. The STRING interaction network for these eleven target proteins was also constructed to study the interactions among them. The interaction network showed 32 edges for eleven nodes with average node degree of 5.82 and ppi enrichment value of 2.85e-09 (Figure 4).

\section{Molecular Docking}

Various clinical and computational studies report the effectiveness of WS extracts as well as its phytocompounds in the control of Oxidative stress. Successfully, all the three withanolides generated good dock poses against the selected target genes with nearly same binding energies for each of the target proteins with minor changes in the binding site and interacting residues (Table 4).

All the three bioactives showed high binding energies against $\mathrm{BCL}_{2}$ protein with binding scores $-9.08,-10.15$ and -9.03 for withanolide A, withaferin A and withanone respectively. The withanolides withanolide $\mathrm{A}$, withaferin $\mathrm{A}$ and withanone showed similar binding energies of $-7.38,-7.37$ and -7.41 with PDGFRB. Withanolide A and withaferin A formed two hydrogen bonds and withanone formed one hydrogen bond with PDGFRB respectively. Withanone showed the highest binding energy with MDM2 with a binding score of -9.23 and interacted with ten residues via alkyl bonds. This was followed up by withanolide A and withaferin $\mathrm{A}$ that interacted with seven and nine residues in the binding pocket and showed binding score of -9.01 and -8.62 respectively. Whereas only withaferin A formed a single hydrogen bond with GLN A: 72 residue of MDM2.

Withaferin A showed the highest binding energy with three hydrogen bonds which was also higher compared to withanolide A and withanone with 1 and 2 hydrogen bonds. Withanolide 


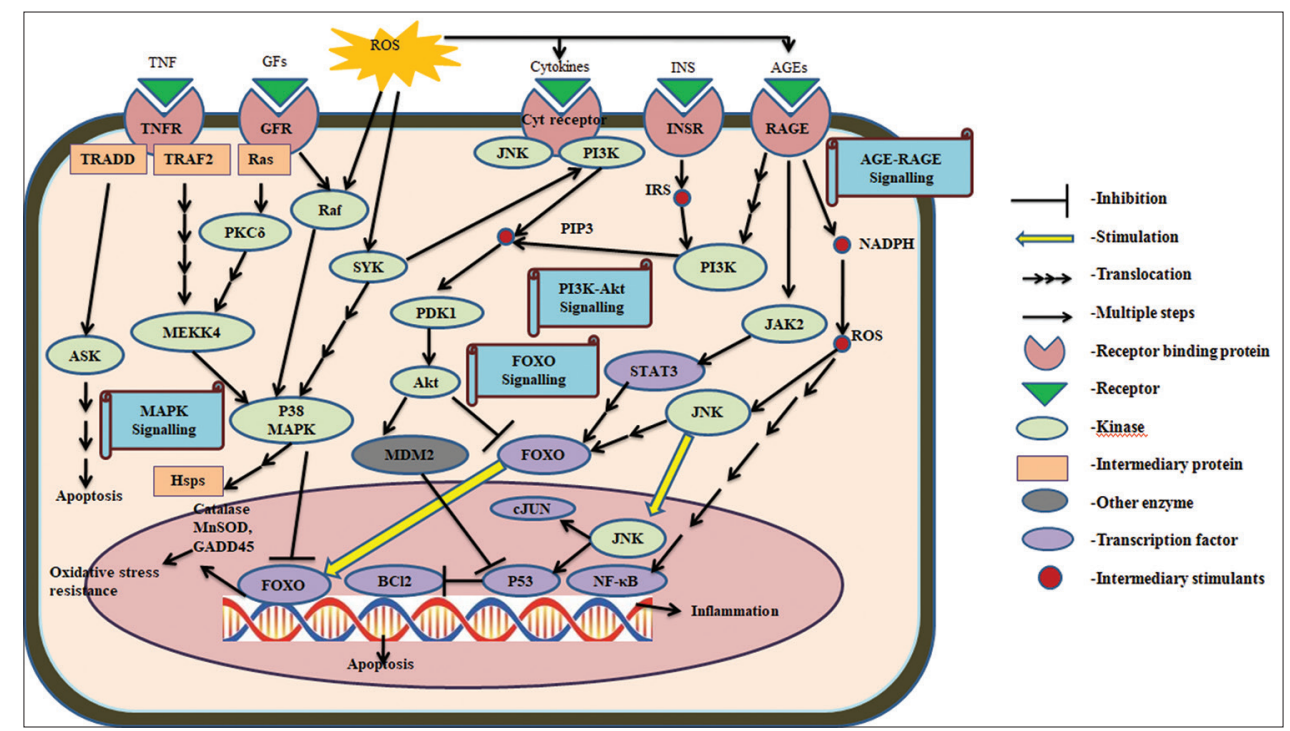

Figure 3: Interplay between the four oxidative stress related pathways

TNF: Tumor necrosis factor; TNFR: Tumor necrosis factor receptor; GFs: Growth factor; GFR: Growth factor receptor; INS: Insulin; INSR: Insulin receptor; AGEs: Advanced glycation end products; RAGE: Advanced glycation end product receptor; TRADD: TNFR1 associated death domain protein; TRAF2: TNFR associated factor 2; ASK: Aspartate kinase; PKC $\delta$ : Serine/threonine protein kinase C delta; MEKK4: Mitogen activated protein kinase kinase 4; P38 MAPK: Mitogen activated protein kinase; MDM2: Mouse double minute 2 homology; Akt: Protein kinase B; SYK: Spleen associated tyrosine kinase; Raf \&Ras: regulators of MAPK pathway; JNK: c-JUN N-terminal kinase; PI3K: phosphor inositol 3 kinase; PDK1: 3- phosphoinositide-dependent kinase 1; JAK2: Janus kinase 2; STAT3: Signal transducer and activator of transcription 3; FOXO: Forkhead box transcription factor; cJUN: Jun proto oncogene; NF-кB: Nuclear factor kappa B; Bcl2: B-cell lymphoma 2; IRS: Insulin receptor substrate 1; PIP3:Phosphatidylinositol 3,4,5 triphosphate; NADPH: Nicotinamide adenine dinucleotide phosphate; ROS: Reactive oxygen species

A, withaferin A and withanone showed binding scores of -6.79 , -6.39 and -6.66 against FOXO1 with 3(ALA F:159, TYR F:165, ASN F:216), 4 (ARG F:180, GLU F:178, SER C:234, GLY C:232) and 3(GLU F:229, ASN C:228, GLN F:185) hydrogen bonds respectively. Withaferin A strongly bound with JAK2 with a binding energy of -9.51 mediated by four hydrogen bonds. The interacting residues were ARG A: 715, PRO A: 700, ASN A: 673 and SER A: 633. Withanone and withanolide A showed binding energies of -9.15 and -8.74 with 1 (THR A: 555) and 2(SER A: 633 and LYS A: 581) hydrogen bonds respectively with JAK2. SYK showed binding energy of -9.19 forming three hydrogen bonds with GLU A: 452, GLU A: 420 and ASP A: 512. Withanolide A and withanone formed l(LEU A: 453) and 2 (ALA A: 451 and ASP A: 512) hydrogen bonds with SYK with binding energies -8.85 and -8.11 respectively. All the three bioactives formed 2 hydrogen bonds with JUN at different binding pockets. Withaferin A with the least binding energy against JUN interacted only with the A chain while withanolide $A$ and withanone interacted with residues of both $A$ and $B$ chain of JUN. Withanolide A, withaferin A and withanone showed binding scores of 6.46, -6.16 and -6.52 against JUN respectively. All the three withanolides showed increased hydrogen bonding with PRKCD compared to the other proteins. PRKCD formed 4,3 and 6 hydrogen bonds with withanolide A, withaferin A and withanone with binding energies $-8.12,-7.37$ and -7.71 respectively.

Withaferin A and withanone formed four hydrogen bonds with different residues of MAPKl contributing to a binding score of -8.51 and -9.43 respectively. Whereas withanolide A formed a single hydrogen bond with GLU A: 33 residue of MAPKl with binding energy -8.68. The highest binding energy with MAPK8 was exhibited by withanone which hydrogen bonded with LYS A: 336 and ASN A: 81 residues with binding energy -9.13. Withaferin A with binding score -8.72 also formed two hydrogen bonds with SER A: 34 and MET A: 111 residues of MAPK8. Withanolide A with three hydrogen bonds showed a docking score of -8.99 against MAPK8.

\section{DISCUSSION}

The adaptogenic potential of Withania somnifera as well as its withanolides can be used to control each stage in the pathophysiology of COVID-19 from the viral entry through immune suppression and cytokine production, inflammation and vascular permeabilisation to multi organ failure (Saggam et al., 2021). The metabolic capability of the drug compound is an important factor to be considered in drug discovery. The in silico ADMET studies on withaferin A, withanolide A and withanone showed no violations against lipinski's rule of five revealing the drug likeliness of all the three withanolides of interest. But in order to understand the mechanism of action of these individual withanolides or its combinations as in crude extracts, the interpretation of the biological targets and associated pathways of these drug compounds becomes a necessity. Though each of the withanolide showed around 300 protein targets, increased overlapping of the targets were observed which might be because 


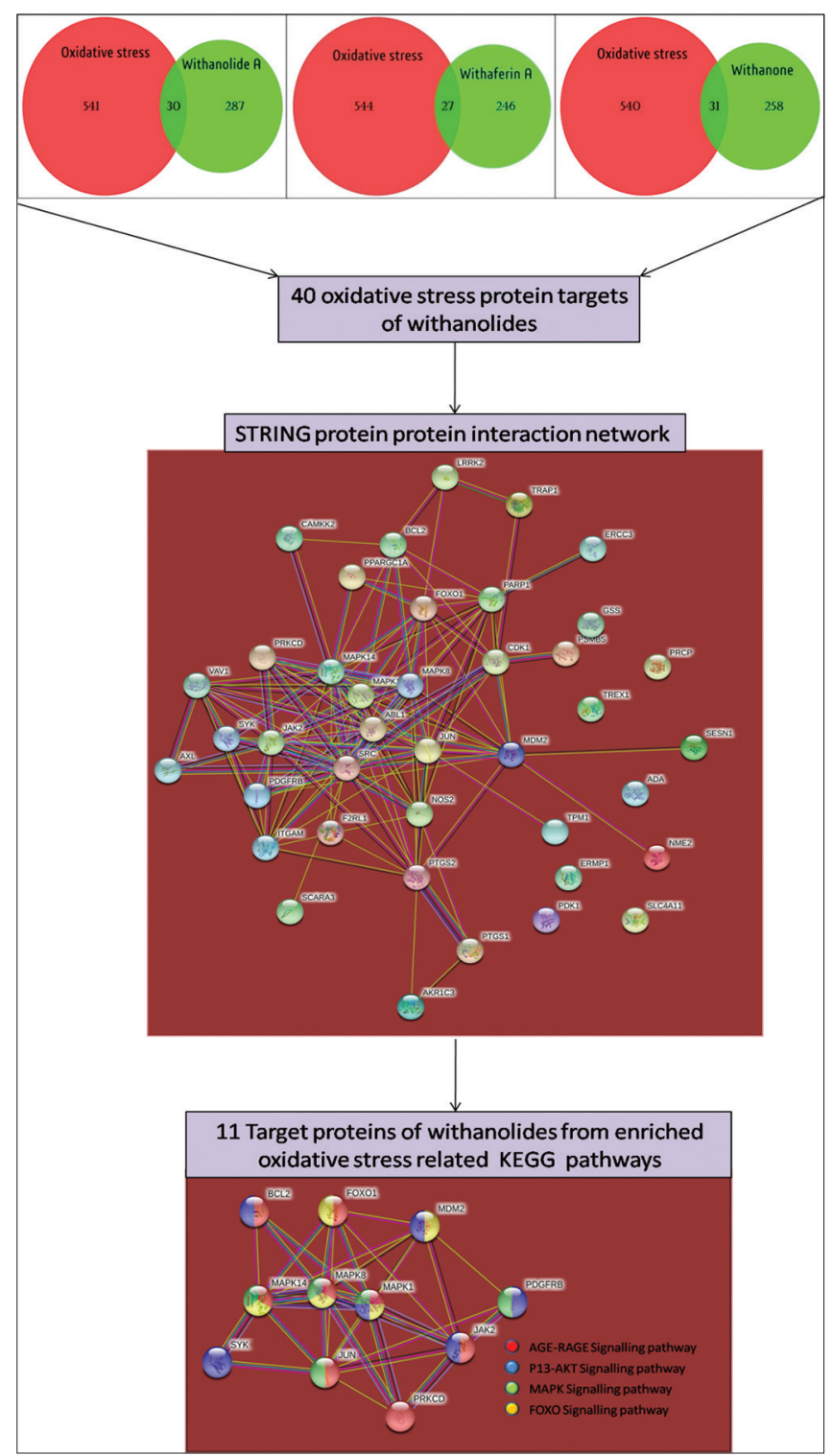

Figure 4: Protein-protein interaction network of the oxidative stress related enriched pathways of the withanolide targets

all the withanolides are structurally similar with slight changes in the side chain or functional groups.

Since oxidative stress remains as a main cause of increased morbidity in COVID 19 cases, the pathways underlying the oxidative stress mechanism namely AGE RAGE signalling pathway, MAPK signalling pathway, PI3-AKT signalling pathway and FOXO signalling pathway were prioritised among the various enriched pathways. It was interesting to note that a similar study on the key target proteins and signalling pathways of COVID-19 also reported 517 target proteins enriched in 32 signalling pathways including the above four pathways (Oh et al., 2021). Thus implicating the anti- COVID potential of the withanolides. Further AGE-RAGE signalling pathway is also reported to play an important role in COVID-19 infection as well as other comorbidities like diabetes, cardiovascular disease, cancer and GI disease that are associated with severity of COVID infection (Chakrabarty et al., 2021).
The protein-protein interaction and pathway enrichment analysis showed that AGE-RAGE signalling pathway was highly enriched among all other pathways associated with the target proteins. It is to be noted that though there are various sources of ROS in a biological system the mitochondrial oxidation and glycation processes remains the primary source. The glycation process leads to the accumulation of glycation end products (AGE) and its interaction with its receptors (RAGE) leads to production of ROS and cytokines via the AGE-RAGE signalling pathway. Among the two forms of RAGE protein, the soluble form (sRAGE) forms the extracellular domain that traps and prevents the binding of ligands to the membrane bound form (mRAGE), thus preventing the inflammatory reactions. The concentrations of sRAGE in the young asymptomatic COVID-19 patients were relatively higher than in non-COVID subjects. Whereas, the elder COVID-19 patients with severe lung injuries showed decreased sRAGE concentrations than in the control subjects (Kehribar et al., 2021). This implies the role of AGE-RAGE pathway in aging and its implications on COVID-19. Among the eleven targets studied, eight target proteins namely BCL2, FOXO1, JUN, PRKCD, JAK2, MAPK1, MAPK8 and MAPK14 were found to be associated with AGERAGE signalling. Though all the three withanolides have shown good docking scores against these targets, the exact role of these withanolides on these genes as well the AGE-RAGE pathway is unknown. But from the experimental studies that have shown anti glycation activity of the withanolides and ashwagandha extracts it can be inferred that these withanolides bind well to these targets thus inhibiting glycation activity which in turn inhibits increased ROS production (Tupe et al., 2016).

The mitogen activated protein kinases (MAPK) also serves to be a component of AGE-RAGE signalling pathway. Several studies have also implicated the role of AGEs in the production of intracellular ROS and also the involvement of MAPK pathway and AKT pathway in AGE -RAGE signalling (Younessi \& Yoonessi, 2011). Mitogen activated protein kinase (MAPK) cascade also referred to as extracellular signal regulated protein kinase (ERK) cascade play an important role in signal transmission by growth factors, immune modulators, cellular stress and other stimuli (Bardwell et al., 2009). The upregulation of p38 MAPK pathway was also observed in COVID-19 patients leading to inflammation and severe organ damage (Grimes \& Grimes., 2020). Under normal conditions, AKT pathway is known to stabilise the homeostasis of the cells by regulating the oxidative metabolism leading to the generation of ROS on one hand and on the other hand suppresses FOXO pathway leading to the transcriptional activation of antioxidant enzymes. This mechanism plays an important role in maintaining the homeostasis of the biological system. The oxidative stress condition also leads to the phosphorylation and translocation of FOXO proteins to the nucleus. Stress activated FOXO proteins induce the expression of tumour suppressor genes leading to cell cycle arrest (Yoko et al., 2005). The activation of FOXO also regulates the immune (innate and adaptive) and inflammatory responses. The diminished anti-oxidant and anti-inflammatory responses in COVID-19 infected patients are the underlying cause for lung injury. The upregulation of FOXO balances 
Table 4 : Molecular docking of the withanolides against the oxidative stress targets

\begin{tabular}{|c|c|c|c|}
\hline Protein & igand & $\begin{array}{l}\text { Binding } \\
\text { Score }\end{array}$ & Interacting residues \\
\hline \multirow[t]{3}{*}{$\overline{B C L 2}$} & Withanolide A & -9.08 & ARG A: 139, LEU A: 137, MET A: 115, ALA A: 149, PHE A: 153, PHE A: 104, VAL A: 133 \\
\hline & Withaferin A & -10.15 & ASP A: 140, GLU A: 136, LEU A: 137, VAL A: 133, P \\
\hline & Withanone & -9.03 & GLU A: 136, ARG A: 139, LEU A: 137, VAL A: 133, MET A: 115, ALA 1:149, PHE A: 153, PHE A: 104 \\
\hline \multirow[t]{3}{*}{ F0X01 } & Withanolide A & -6.79 & ALA F: 159, TYR F: 196, TRP F: 160, TYR F: 165, ASN F: 216, ALA F: 166, HIS F: 220, SER F: 164 \\
\hline & Withaferin A & -6.39 & TRP F: 236, GLU C: 229, ARG F: 180, GLU F: 178, TRP C: 236, LYS C: 233, SER C: 234, GLY C: 232 \\
\hline & Withanone & -6.66 & GLU F: 229, ASN C: 228, LYS F: 233, TRP F: 236, GLN F: 185, LYS C: 233 \\
\hline \multirow[t]{3}{*}{ MDM2 } & Withanolide A & -9.01 & TYR A: 100, HIS A: 96, LEU A: 54, VAL A: 93, ILE A: 61, MET A: 62, TYR A: 67 \\
\hline & Withaferin A & -8.62 & PHE A: 55, ILE A: 61, VAL A: 93, VAL A: 75, TYR A: 67, GLN A: 72, LEU A: 54, \\
\hline & Withanone & -9.23 & $\begin{array}{l}\text { ILE A: 61, VAL A: 93, LEU A: 57, HIS A: 96, ILE A: 99, TYR A: 100, LEU A: 54, GLY A: 58, MET A: 62, TYR } \\
\text { A: } 67\end{array}$ \\
\hline \multirow[t]{3}{*}{ JUN } & e A & -6.46 & LYS A: 288, GLN B: 290, ARG A: 302, LEU B: 294, GLU B: 293 \\
\hline & A & -6.16 & TYR B: 315, ASN B: 314, MET B: 313, VAL B: 312 \\
\hline & Withanone & -6.52 & LYS B: 309, VAL B: 305, LEU A: 301, ARG B: 302, MET A: 300, THR A: 297 \\
\hline \multirow[t]{3}{*}{ JAK2 } & Withanolide A & -8.74 & PHE A: 628, LEU A: 579, LEU: 551, LYS A: 581, SER A: 698, SER A: 633, ILE A: 559 \\
\hline & Withaferin A & -9.51 & $\begin{array}{l}\text { ARG A: 715, PRO A: 700, ASN A: 673, LEU A: 680, ILE A: 559, LEU A: 551, SER A: 633, LYS A: 677, THR A: } \\
555\end{array}$ \\
\hline & Withanone & -9.15 & $\begin{array}{l}\text { LEU A: 551, LYS A: 581, PHE A: 620, LEU A: 579, ILE A: 559, LYS A: 677, ASN A: 678, GLY A: 554, THR A: } \\
555\end{array}$ \\
\hline \multirow[t]{3}{*}{ SYK } & Withanolide A & -8.85 & $\begin{array}{l}\text { VAL A: 385, ALA A: 400, MET A: 450, LEU A: 501, LYS A: 458, PRO A: 455, GLN A: 462, TYR A: 459, LEU A: } \\
453\end{array}$ \\
\hline & Withaferin A & -9.19 & $\begin{array}{l}\text { GLU A: 452, MET A: 450, LEU A: 377, ALA A: 400, LYS A: 402, GLU A: 420, ASP A: 512, SER A: 511, PRO A: } \\
\text { 455, LEU A: } 501\end{array}$ \\
\hline & Withanone & -8.11 & $\begin{array}{l}\text { LEU A: 377, ALA A: 451, ALA A: 400, GLU A: 452, MET A: 450, VAL A: 385, LEU A: 501, SER A: 511, MET } \\
\text { A: 448, LYS A: 402, ASP A: } 512\end{array}$ \\
\hline \multirow[t]{3}{*}{ PDGFRB } & Withanolide A & -7.38 & LEU A: 28, TRP A: 32, LYS A: 35, ARG A: 37, LEU A: 31, TYR A: 38 \\
\hline & With & -7.37 & MET B: 30, ILE A: $27, \mathrm{~S}$ \\
\hline & Withe & -7.41 & ILE B: 26, ILE B: 29, LY \\
\hline \multirow[t]{3}{*}{ MAPK1 } & Withanolide A & -8.68 & GLU A: 33, VAL A: 39, LYS A: 114, ILE A: 31, ILE \\
\hline & Withaferin A & -8.51 & , ASP A: 88 , ILE A: 89, GLN \\
\hline & Witha & -9.43 & 4, ARG A: 353, LEU A: 76, ARG A \\
\hline \multirow[t]{3}{*}{ MAPK8 } & Withanolide A & -8.99 & LYS A: 300, ILE A: 310, LEU A: 241, LEU A: 302, ILE A: 304, ASP A: 305, VAL A: 303 \\
\hline & Withaferin A & -8.72 & 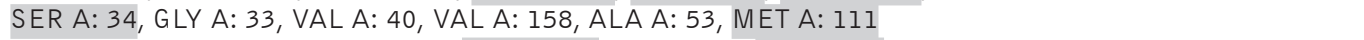 \\
\hline & Withanone & -9.13 & HIS A: 82, ALA A: 332, PRO A: 335, LYS A: 336, C \\
\hline \multirow[t]{3}{*}{ MAPK14 } & Withanolide A & -9.51 & $\begin{array}{l}\text { TYR A: 35, VAL A: 38, ALA A: 51, PHE A: 169, LYS A: 53, LEU A: 55, SER A: 56, HIS A: 64, THR A: 68, ARG } \\
\text { A: } 67\end{array}$ \\
\hline & With & -9.02 & LEU A: 75, LEU A: 74, ARG A: 149, HIS A: \\
\hline & Witha & -9.46 & $\begin{array}{l}\text { LEU A: 55, GLU A: 71, HIS A: 64, ARG A: 67, LEU A: 104, LYS A: 53, ALA A: 51, VAL A: 38, PHE A: 169, } \\
\text { SER A: } 56\end{array}$ \\
\hline \multirow[t]{3}{*}{ PRKCD } & Withanolide A & -8.12 & PHE A: 243, THR A: 242, SER A: 240, ASN A: 267, LYS A: 260, TYR A: 236 \\
\hline & Withaferin A & -7.37 & $\begin{array}{l}\text { TYR A: 236, MET A: 239, TYR A: 238, SER A: 240, PRO A: 241, PHE A: 243, ASP A: 245, CYS A: 244, GLY A: } \\
\text { 248, THR A: 242, LYS A: 260, ASN A: } 267\end{array}$ \\
\hline & Withanone & -7.71 & PHE A: 243, THR A: 242, SER A: 240, MET A: 239, LYS A: 260, TYR A: 236, ASN A: 267 \\
\hline
\end{tabular}

The residues forming hydrogen bonds with the withanolides are highlighted in grey

the concentration of pro-inflammatory molecules thus aiding repair of the damaged tissues (Cheema et al., 2021).

FOXO transcription factors are also negatively regulated by AKT activation. Both withanolide $\mathrm{A}$ and withaferin $\mathrm{A}$ are found to inhibit AKT pathway at certain concentrations thus leading to the activation of FOXO transcription (Wang et al., 2019; Yan et al., 2018). The down regulation of AKT pathway leads to reduced ROS levels due to decreased mitochondrial activity and increased FOXO mediated antioxidant enzyme production (Dolado \& Nebreda, 2008). Besides activation of FOXO by phosphorylation of the upstream region by AKT, it was found that in mammals, any extracellular signal that activates the AKT pathway is also found to directly inhibit the FOXO transcription factors. The upregulation of antioxidant enzymes and suppression of oxidative stress by withaferin A was inhibited by the addition of AKT inhibitor IV indicating its inhibitory role on AKT pathway (Yan et al., 2018).
Withanolide A was also found to inhibit AKT pathway at higher concentrations but activated the same at lower concentrations (Wang et al., 2019).

The grid based Molecular docking simulation studies were carried out to study the binding potential of the withanolides against the anti-stress target proteins. Hydrogen bonds also play a key role in determining the strength of the intermolecular interactions as well as the three dimensional conformation adopted by the proteins. Further, the docking score differentiates the interacting ability of the compounds. Though the bioactives selected for the study are structurally similar, they show significantly different activities making them ideal candidates to be investigated through virtual screening and docking. Various studies have reported the antioxidant capability of anti-apoptotic protein BCL2 like the suppression of hyperglycemia-induced formation of advanced glycation end products (AGE) in endothelial cells and reduced ROS 
generation in glutathione depleted cells (Susnow et al., 2009). The ROS mediated activation of MAPK pathway also suppresses the BCL2 expression in the cells (Changchien et al., 2015). The decreased levels of BCL2 were observed in COVID-19 mortality cases than in COVID-19 survivors (Lorente et al., 2021). On the other hand, withaferin A pretreatment has shown the reversal of BCL2 downregulation in bromobenzene induced mice models accompanied by decrease in the levels of inflammatory cytokines TNF $\alpha$ and IL1 $\beta$ along with the restoration of antioxidant enzymes levels that were suppressed by bromobenzene (Vedi \& Sabina, 2016). These studies implicate the upregulation of BCL2 protein by withaferin A to be used against COVID-19 severity. Withanone has also shown to reduce NMDA (N-methyl D-aspartate) induced ROS production and maintained Bax/ BCL2 ratio significantly in neuron like cells (Dolado \& Nebreda, 2008). All the three withanolides expressed higher binding score towards BCL2 compared to all the other target genes.

Once inside the cells, viral components directly activate a series of signalling pathways leading to the post translational modification of $\mathrm{P} 53$ along with its negative regulator MDM2. The loss of MDM2 function leads to P53 mediated induction of viral growth by decreasing cell apoptosis as in viral infections like SARS-CoV, MERS-CoV, and SARSCoV-2 PLP (Zauli et al., 2020). Thus MDM2 can also be regarded as a potential target anti antiviral drugs. The docking poses of the three bioactives with MDM2 shows the occupation of the same binding pocket but with a slight difference in orientation of the withanolides within the binding region. Like most of the growth factor receptors, Platelet derived growth factor receptor (PDGFRB) are known to play an important role in the progression of viral infections right from adhering the cell surface to altering the signalling pathways to aid in replication (Vrijens et al., 2019). Thus compounds targeting the PDGFRB can be used as potent anti viral drugs. All the three withanolides were found to interact with the PDGFRB protein with withanolide A showing maximum interaction. The anti-inflammatory potential of FOXO transcription factors along with its role in maintaining normal cell physiology, regulating oxidative stress and immunity has turned the focus of researches for its role in viral infections. FOXO activation is reported to decrease the cytokine storm which is the main cause of mortality in COVID-19 patients and also produce immunological memory (Cheema et al., 2021). The studies on the genes involved in COVID-19 infection and its role in the underlying comorbidities revealed JAK2 as the core hub gene in cardiovascular disease, hypertension as well as kidney disorders (Barh et al., 2021). Therefore drugs targeting JAK2 can be used in COVID-19 patients with any of these underlying diseases. JAK2, along with SYK, JUN and PRKCD were present as the top 10 hub genes common in COVID-19 infection and Kidney disorders (Barh et al., 2021). Protein kinase C-delta (PKC- $\delta$ or PRKCD) are known to be phosphorylated at TYR 512 and TYR 523 in response to $\mathrm{H}_{2} \mathrm{O}_{2}$ induced oxidative stress. This activation of the protein PRKCD leads to its translocation from the cytoplasm to the mitochondria which is required to bring about the apoptotic response of the cells under oxidative stress (Majumder et al., 2001). MAPKl and
MAPK8 were among the seven core gene set that were shared among diabetic pathways and COVID infection. Thus can act as suitable target for COVID-19 patients with diabetes. All the withanolides studied showed good scores against FOXO, JAK2 and JUN. Further MAPK14 was recognised as a protein for COVID patients with underlying, heart and kidney disorders (Barh et al., 2021). The pathway enrichment showed the association of MAPK with all the four selected pathways. Further all the three bioactives showed good docking conformations at different binding sites of MAPK1, MAPK8 and MAPK14.

\section{CONCLUSION}

Withania somnifera owing to its pharmacological activity has been evaluated against various diseases in various models in vitro and in vivo. But the role of individual metabolites of this plant on different pathways underlying the diseases has not been much explored. The present study is a computational approach to identify the possible pathway modulation by the withanolides to overcome oxidative stress. The above results show that all the three withanolides pocess nearly same binding energies with little similarity in the binding pockets and interacting residues. Thus these withanolides can be considered as a potential therapeutic compound for chronic diseases as it modulate several signalling pathways. But the combination of multiple components as in plant extracts or formulations may show synergistic activities to overcome stress in a better way than the individual lead hit. Further in vitro and in vivo examinations have to be conducted to study the potential activity of the individual withanolides as well as its combined activity when administered as plant extracts and formulations to overcome these pandemic diseases.

\section{REFERENCES}

Ahmad, M. K., Mahdi, A. A., Shukla, K. K., Islam, N., Rajender, S., Madhukar, D., Shankhwar, S. N., \& Ahmad, S. (2010). Withania somnifera improves semen quality by regulating reproductive hormone levels and oxidative stress in seminal plasma of infertile males. Fertility and Sterility, 94, 989-996. https://doi.org/10.1016/j. fertnstert.2009.04.046

Aswathy, L., Jisha, R. S., Masand, V. H., Gajbhiye, J. M., \& Shibi, I. G. (2018). Design of novel amyloid $\beta$ aggregation inhibitors using QSAR, pharmacophore modeling, molecular docking and ADME prediction. In Silico Pharmacology, 6(1), 12. https://doi.org/10.1007/ s40203-018-0049-1

Bakadia, B. M., Boni, B., Ahmed, A., \& Yang, G. (2021). The impact of oxidative stress damage induced by the environmental stressors on COVID-19. Life sciences, 264, 118653. https://doi.org/10.1016/j. Ifs. 2020.118653

Bardwell, A. J., Frankson, E., \& Bardwell, L. (2009). Selectivity of docking sites in MAPK kinases. Journal of Biological Chemistry, 284(19), 13165-13173. https://doi.org/10.1074/jbc.M900080200

Barh, D., Aljabali, A. A., Tambuwala, M. M., Tiwari, S., Serrano-Aroca, Á. Alzahrani, K. J., Andrade, B. S., Azevedo, V., Ganguly, N. K., \& Lundstrom, K. (2021). Predicting COVID-19-Comorbidity Pathway Crosstalk-Based Targets and Drugs: Towards Personalized COVID-19 Management. Biomedicines, 9(5), 556. https://doi.org/10.3390/ biomedicines9050556

Bhattacharya, S. K., \& Muruganandam, A. V. (2003). Adaptogenic activity of Withania somnifera: An experimental study using a rat model of chronic stress. Pharmacology, Biochemistry, and Behavior, 75(3), 547-555. https://doi.org/10.1016/S0091-3057(03)00110-2 
Carbon, S., Ireland, A., Mungall, C. J., Shu, S., Marshall, B., Lewis, S., Lomax, J., Mungall, C., Hitz, B., Balakrishnan, R., Dolan, M., Wood, V., Hong, E., \& Gaudet, P. (2009). AmiGO: Online access to ontology and annotation data. Bioinformatics, 25(2), 288-289. https://doi. org/10.1093/bioinformatics/btn615

Chakrabarty, B., Das, D., Bulusu, G., \& Roy, A. (2021). Network-Based Analysis of Fatal Comorbidities of COVID-19 and Potential Therapeutics. IEEE/ACM transactions on Computational Biology and Bioinformatics, 18(4), 1271-1280. https://doi.org/10.1109/ tcbb.2021.3075299

Chandel, V., Raj, S., Rathi, B., \& Kumar, D. (2020). In silico identification of potent fda approved drugs against coronavirus covid-19 main protease: A drug repurposing approach. Chemical Biology Letters, 7(3), 166-175.

Changchien, J. J., Chen, Y. J., Huang, C. H., Cheng, T. L., Lin, S. R., \& Chang, L. S. (2015). Quinacrine induces apoptosis in human leukemia K562 cells via p38 MAPK-elicited BCL2 down-regulation and suppression of ERK/c-Jun-mediated BCL2L1 expression. Toxicology and Applied Pharmacology, 284(1), 33-41. https://doi.org/10.1016/j. taap.2015.02.005

Cheema, P. S., Nandi, D., \& Nag, A. (2021). Exploring the therapeutic potential of forkhead box O for outfoxing COVID-19. Open Biology, 11(6), 210069 https://doi.org/10.1098/rsob.210069

Chernyak, B. V. Popova, E. N., Prikhodko, A. S., Grebenchikov, O. A., Zinovkina, L. A., \& Zinovkin, R. A. (2020). COVID 19 and Oxidative Stress. Biochemistry, 85(12), 1543-1553. https://doi.org/10.1134/ S0006297920120068

Daina, A., Michielin, O., \& Zoete, V. (2019). SwissTargetPrediction: updated data and new features for efficient prediction of protein targets of small molecules. Nucleic Acids Research, 47, W357-W3664. https:// doi.org/10.1093/nar/gkz382

Dhawan, M., Parmar, M., Sharun, K., Tiwari, R., Bilal, M. \& Dhama, K. 2021. Medicinal and therapeutic potential of withanolides from Withania somnifera against COVID-19. Journal of Applied Pharmaceutical Science, 11(4), 006-013. https://doi.org/10.7324/JAPS.2021.110402

Dolado, I., \& Nebreda, A. R. (2008). AKT and Oxidative Stress Team Up to Kill Cancer Cells. Cancer Cell, 14(6), 427-429. https://doi.org/10.1016/j. ccr.2008.11.006

Farías, J. G., Molina, V. M., Carrasco, R. A., Zepeda, A. B., Figueroa, E., Letelier, P., \& Castillo, R. L. (2017). Antioxidant therapeutic strategies for cardiovascular conditions associated with oxidative stress. Nutrients, 9(9), 966. https://doi.org/10.3390/nu9090966

Fonseka, P., Pathan, M., Chitti, S. V., Kang, T., \& Mathivanan, S. (2021). FunRich enables enrichment analysis of OMICs datasets. Journal of Molecular Biology, 433(11), 166747. https://doi.org/10.1016/j. jmb.2020.166747

Grimes, J. M., \& Grimes, K. V. (2020). p38 MAPK inhibition: A promising therapeutic approach for COVID-19. Journal of Molecular and Cellular Cardiology, 144, 63-65. https://doi.org/10.1016/j.yjmcc.2020.05.007

Kehribar, D. Y., Cihangiroglu, M., Sehmen, E., Avci, B., Capraz, A., Bilgin, A.Y., Gunaydin, C., \& Ozgen, M. (2021). The receptor for advanced glycation end product (RAGE) pathway in COVID-19. Biomarkers, 26(2), 114-118. https://doi.org/10.1080/135475 OX.2020.1861099

Khanal, P., Patil, B. M., Chand, J., \& Naaz, Y.(2020). Anthraquinone Derivatives as an Immune Booster and their Therapeutic Option Against COVID-19. Natural Products and Bioprospecting, 10(5), 325-335. https://doi.org/10.1007/s13659-020-00260-2

Koval, L., Zemskaya, N., Aliper, A., Zhavoronkov, A., \& Moskalev, A. (2021). Evaluation of the geroprotective effects of withaferin A in Drosophila melanogaster. Aging, 13(1), 1817-1841. https://doi.org/10.18632/ aging. 202572

Kumar, V., Dhanjal, J. K., Kaul, S. C., Wadhwa, R., \& Sundar, D. (2020). Withanone and caffeic acid phenethyl ester are predicted to interact with main protease (Mpro) of SARS-CoV-2 and inhibit its activity. Journal of Biomolecular Structure and Dynamics, 39(11), 3842-3854. https://doi.org/10.1080/07391102.2020.1772108

Lohidashan, K., Rajan, M., Ganesh, A., Paul, M., \& Jerin, J. (2018). Pass and Swiss ADME collaborated in silico docking approach to the synthesis of certain pyrazoline spacer compounds for dihydrofolate reductase inhibition and antimalarial activity. Bangladesh Journal of Pharmacology, 13(1), 23-29. https://doi.org/10.3329/bjp.v13i1.33625

Lorente, L., Martín, M. M., González-rivero, A. F., Argueso, M., Perez, A., Ramos-gómez, L., Solé-, J., Alberto, J., Ramos, M. Y., Ojeda, N.,
Jiménez, A., Lorente, L., Martín, M. M., González-rivero, A. F., Argueso, M., Perez, A., Ramos-gómez, L., Solé-violán, J., Alberto, J., Ramos, M. Y., Ojeda, N., \& Jiménez, A. (2021). Expert Review of Molecular Diagnostics Blood concentrations of proapoptotic sFas and antiapoptotic Bcl2 and COVID-19 patient mortality. Expert Review of Molecular Diagnostics, 21(7), 837-844. https://doi.org/10.1080/1 4737159.2021.1941880

Majumder, P. K., Mishra, N. C., Sun, X., Bharti, A., Kharbanda, S., Saxena, S., \& Kufe, D. (2001). Targeting of protein kinase $C \delta$ to mitochondria in the oxidative stress response. Cell Growth and Differentiation, 12(9), 465-470.

Masuda, T., Shimazawa, M., \& Hara, H. (2017). Retinal Diseases Associated with Oxidative Stress and the Effects of a Free Radical Scavenger (Edaravone). Oxidative Medicine and Cellular Longevity, 9208489. https://doi.org/10.1155/2017/9208489

Matsuoka, H. (2001). Endothelial dysfunction associated with oxidative stress in human. Diabetes Research and Clinical Practice, 54, S65S72. https://doi.org/10.1016/S0168-8227(01)00337-0

Muhammad, Y., Kani, Y. A., Iliya, S., Muhammad, J. B., Binji, A. Ahmad, A. E., Kabir, M. B., Bindawa, K. U \& Ahmed, Y. (2021) Deficiency of antioxidants and increased oxidative stress in COVID-19 patients : A cross-sectional comparative study in Jigawa, Northwestern Nigeria. SAGE Open Medicine, 9, 2050312121991246. https://doi.org/10.1177/2050312121991246

Nab, J., Abdelfatah, S., \& Efferth, T. (2021). Induction of stress resistance and extension of lifespan in Chaenorhabditis elegans serotoninreceptor knockout strains by withanolide A. Phytomedicine, 84 153482. https://doi.org/10.1016/j.phymed.2021.153482

Oh, K., Adnan, M., \& Cho, D. (2021). Drug-repurposing against COVID-19 by targeting a key signaling pathway: An in silico study. Medical Hypotheses, 155, 110656. https://doi.org/10.1016/j. mehy.2021.110656

Palipoch, S. (2013). A review of oxidative stress in acute kidney injury: protective role of medicinal plants-derived antioxidants. African Journal of Tradittional, Complementary, and Alternative Medicines, 10(4), 88-93

Pawlak, K., Domaniewski, T., Mysliwiec, M., \& Pawlak, D. (2009). The kynurenines are associated with oxidative stress, inflammation and the prevalence of cardiovascular disease in patients with endstage renal disease. Atherosclerosis, 204(1), 309-314. https://doi. org/10.1016/j.atherosclerosis.2008.08.014

Prasad, S., Srivastava, S. K. (2020). Oxidative stress and cancer: Chemopreventive and therapeutic role of triphala. Antioxidants, 9(1) 72. https://doi.org/10.3390/antiox9010072

Rajkumar, R. P. (2020). Ayurveda and COVID-19: Where psychoneuroimmunology and the meaning response meet. Brain, Behavior, and Immunity, 87, 8-9. https://doi.org/10.1016/j. bbi.2020.04.056

Saggam, A., Limgaokar, K., Borse, S., Chavan-gautam, P., \& Dixit, S. (2021) Withania somnifera (L.) Dunal : Opportunity for Clinical Repurposing in COVID-19. Management. Frontiers in Pharmacology, 12, 623795 https://doi.org/10.3389/fphar.2021.623795

Shah, N., Singh, R., Sarangi, U., Saxena, N., Chaudhary, A., Kaur, G., Kaul, S., \& Wadhwa, R. (2015). Combinations of Ashwagandha leaf extracts protect brain-derived cells against oxidative stress and induce differentiation. PloS One, 10(3), e0120554. https://doi.org/10.1371/ journal.pone.0120554

Susnow, N., Zeng, L., Margineantu, D., \& Hockenbery, D. M. (2009). $\mathrm{Bcl}-2$ family proteins as regulators of oxidative stress. Seminars in Cancer Biology, 19(1), 42-49. https://doi.org/10.1016/j. semcancer.2008.12.002

Szklarczyk, D., Gable, A. L., Lyon, D., Junge, A., Wyder, S., Huerta-Cepas, J., Simonovic, M., Doncheva, N. T., Morris, J. H., Bork, P., Jensen, L. J., \& Von Mering, C. (2019). STRING v11: Protein-protein association networks with increased coverage, supporting functional discovery in genome-wide experimental datasets. Nucleic Acids Research, 47(D1), D607-D613. https://doi.org/10.1093/nar/gky1131

Tupe, R. S., Kemse, N. G., Khaire, A. A., \& Shaikh, S. A. (2016). Attenuation of glycation- induced multiple protein modifications by Indian antidiabetic plant extracts. Pharmaceutical Biology, 55(1), 68-75. https://doi.org/10.1080/13880209.2016.1228683

Vedi, M., \& Sabina, E. P. (2016). Assessment of hepatoprotective and nephroprotective potential of withaferin A on bromobenzene-induced injury in Swiss albino mice: possible involvement of mitochondrial 
dysfunction and inflammation. Cell Biology and Toxicology, 32(5), 373-390. https://doi.org/10.1007/s10565-016-9340-2

Vrijens, P., Noppen, S., Boogaerts, T., Vanstreels, E., Ronca, R., Chiodelli, P., Laporte, M., Vanderlinden, E., Liekens, S., Stevaert, A., \& Naesens, L. (2019). Influenza virus entry via the GM3 ganglioside-mediated plateletderived growth factor receptor $\beta$ signalling pathway. Journal of General Virology, 100(4), 583-601. https://doi.org/10.1099/jgv.0.001235

Wang, H. C., Hu, H. H., Chang, F. R., Tsai, J. Y., Kuo, C. Y., Wu, Y. C. \& Wu, C.C. (2019). Different effects of $4 \beta$-hydroxywithanolide $E$ and withaferin A, two withanolides from Solanaceae plants, on the Akt signaling pathway in human breast cancer cells. Phytomedicine, 53, 213-222. https://doi.org/10.1016/j.phymed.2018.09.017

Yan, Z., Guo, R., Gan, L., Lau, W. B., Cao, X., Zhao, J., Ma, X., Christopher, T. A., Lopez, B. L., \& Wang, Y. (2018). Withaferin A inhibits apoptosis via activated Akt-mediated inhibition of oxidative stress. Life Sciences, 211, 91-101. https://doi.org/10.1016/j.Ifs.2018.09.020
Yoko, F., Yosuke, K., \& Chen. (2005). FOXO Transcription Factors in CellCycle Regulation and the Response to Oxidative Stress. Antioxidants \& Redox Signaling, 7(5-6), 752-760. https://doi.org/10.1111/j.14754959.2010.00371.x

Younessi, P., \& Yoonessi, A. (2011). Advanced glycation end-products and their receptor-mediated roles: Inflammation and oxidative stress. Iranian Journal of Medical Sciences, 36(3), 154-166.

Yu, T. J., Tang, J. Y. Ou-Yang, F., Wang, Y. Y., Yuan, S. S. F., Tseng, K. Lin, L. C., \& Chang, H. W. (2020). Low concentration of withaferin a inhibits oxidative stress-mediated migration and invasion in oral cancer cells. Biomolecules, 10(5), 777. https://doi.org/10.3390/ biom 10050777

Zauli, G., Tisato, V., \& Secchiero, P. (2020). Rationale for Considering Oral Idasanutlin as a Therapeutic Option for COVID-19 Patients. Frontiers in Pharmacology, 11, 1156. https://doi.org/10.3389/ fphar.2020.01156 